\title{
Utilidad del ecógrafo portátil en la sala de hemodiálisis para el cambio del tipo de acceso vascular: de catéter venoso tunelizado a fístula arteriovenosa
}

\author{
Raúl Darbas-Barbé, Ramón Roca-Tey, Juan Carlos González Oliva, Celia Balada Sancho, Sara Tornel García, \\ Tania Curado Soto, Laura Román García. \\ Unidad de Diálisis. Área de Nefrología. Hospital de Mollet. Fundación Sanitaria Mollet. Mollet del Vallés. Barcelona. \\ España
}

\section{Resumen}

Objetivo: Determinar la utilidad de la ecografía-doppler vascular en la sala de hemodiálisis, en la identificación de pacientes portadores de catéter venoso tunelizado susceptibles de la construcción de una fístula arteriovenosa.

Material y Método: Estudio descriptivo transversal en pacientes en hemodiálisis crónica portadores de un catéter venoso tunelizado. Se realizaron evaluaciones mediante ecografía portátil modo $B$, en 3 puntos diferentes de ambos brazos (proximal, medio y distal), y sobre 2 puntos de ambos antebrazos (proximal y distal), recogiéndose un promedio de las determinaciones realizadas. Se recogieron parámetros morfológicos cualitativos (presencia/ausencia de calcificación arterial) y cuantitativos (diámetro y profundidad) de la arteria radial, la humeral y la vena basílica de ambas extremidades superiores.

Resultados: De las 63 personas en hemodiálisis, 9 eran portadores de un catéter tunelizado yugular (14,3\%). Los valores medios de diámetro y profundidad $(\mathrm{mm})$ fueron los siguientes: $4,7 \pm 3,0$ y 9,2 $\pm 2,7$ (arteria humeral derecha), $4,8 \pm 0,6$ y $10,5 \pm 3,3$ (arteria humeral izquierda), 2,6 $6 \pm 0,4$ y 6,6 $6 \pm 2,0$ (arteria radial derecha), $2,7 \pm 0,7$ y $6,0 \pm 1,6$ (arteria radial izquierda), $4,3 \pm 0,6$ y $10,1 \pm 2,1$ (vena basílica derecha), $5,1 \pm 0,5$ y $10,1 \pm 2,1$ (vena basílica izquierda). En la mayoría de los casos $(66,7 \%)$ se evidenció ausencia de calcificaciones, una placa aislada de calcificación en un caso $(11,1 \%)$ y más de una en dos casos $(22,2 \%)$.

Conclusión: El ecógrafo portátil utilizado "in situ" en la sala de HD es una exploración útil para conseguir un cambio en el tipo de acceso vascular, es decir, para la conversión de un catéter venoso tunelizado en una fístula arteriovenosa en las personas en hemodiálisis crónica.

PALABRAS CLAVE: ecografía doppler; fístula arteriovenosa; catéter venoso tunelizado; hemodiálisis.

Usefulness of portable ultrasound in the hemodialysis unit for changing the type of vascular access: from tunneled venous catheter to arteriovenous fistula

\section{Abstract}

Objective: To determine the utility of vascular ultrasound/Doppler in the hemodialysis unit about the identification of patients with tunneled venous catheter, who can be candidates for an arteriovenous fistula.

Material and Method: Cross-sectional descriptive study in patients on chronic hemodialysis with tunneled venous catheters. Evaluations were carried out by B mode portable ultrasound, in 3 different points of both arms (proximal, middle and distal), and on 2 points of both forearms (proximal and distal), collecting an average in such determinations. Qualitative morphological parameters (presence/absence of arterial calcification) 
and quantitative parameters (diameter and depth) of the radial, humeral and basilic veins of both upper extremities were collected.

Results: Of the 63 people on hemodialysis, 9 were carriers of a tunnelled jugular catheter $(14.3 \%)$. The mean values of diameter and depth $(\mathrm{mm})$ were the following: 4.7 \pm 3.0 and $9.2 \pm 2.7$ (right humeral artery), $4.8 \pm 0.6$ and $10.5 \pm 3.3$ (left humeral artery), 2.6 \pm 0.4 and $6.6 \pm 2.0$ (right radial artery), $2.7 \pm 0.7$ and $6.0 \pm 1.6$ (left radial artery), $4,3 \pm 0.6$ and $10.1 \pm 2.1$ (right basilic vein), $5.1 \pm 0.5$ and $10.1 \pm 2.1$ (left basilic vein). In the majority of cases $(66.7 \%)$, absence of calcifications was evidenced, an isolated plaque of calcification in one case $(11.1 \%)$ and more than one in two cases $(22.2 \%)$.

Conclusion: The portable ultrasound system used "in situ" in the HD unit is a useful exploration to achieve a change in the type of vascular access, that is, for the conversion of a tunneled venous catheter to an arteriovenous fistula in people with chronic hemodialysis.

KEYWORDS: doppler ultrasound; arteriovenous fistula; tunneled venous catheter; hemodialysis.

\section{Introducción}

De los tres tipos de acceso vascular permanente utilizados en la práctica clínica habitual para efectuar el tratamiento sustitutivo renal mediante hemodiálisis (HD), es decir, la fistula arteriovenosa (FAV) nativa (FAVn), la fístula arteriovenosa protésica (FAVp) y el catéter venoso tunelizado (CVT); se considera a la FAVn como el de elección en la mayoría de los casos debido a que, una vez se ha conseguido su maduración, presenta una mayor permeabilidad y una menor tasa de complicaciones ${ }^{1}$.

Por otra parte, se considera al CVT como la peor de las opciones que debe evitarse siempre que sea posible debido a su morbimortalidad asociada ${ }^{1,2}$. Se ha demostrado que, aplicando un modelo de riesgo competitivo multivariante, iniciar programa de HD mediante un CVT aumenta el riesgo de muerte para todas las causas a lo largo del tiempo en un $55 \%$ respecto a la $\mathrm{FAVn}^{3}$. En este sentido, Gruss et al evidenciaron un riesgo ajustado de mortalidad por todas las causas 1,86 veces mayor para los usuarios que comenzaron HD con un CVT respecto a la $\mathrm{FAVn}$ y demostraron que las personas que necesitaban un CVT presentaron un riesgo ajustado de mortalidad por 1,68 veces superior que aquellas que siempre habían utilizado una $\mathrm{FAVn}^{4}$. Además, este riesgo de mortalidad depende del tiempo de exposición al CVT, de forma que el riesgo ajustado de mortalidad por todas las causas fue 7,66 veces mayor para las personas que fueron dializados con un CVT durante más del $52 \%$ del tiempo de seguimiento en relación con quienes fueron dializados mediante un CVT durante menos del $18 \%$ del tiempo de seguimiento ${ }^{4}$.

Por tanto, hay que minimizar como sea el tiempo de exposición de la persona prevalente al CVT intentando la construcción precoz de una FAV (FAVn o FAVp). Para conseguirlo, la ecografía doppler nos puede proporcionar una ayuda inestimable ${ }^{5}$ : el mapeo ecográfico vascular de ambas extremidades superiores en personas prevalentes dializadas mediante un CVT constituye una herramienta indispensable para evaluar las posibilidades reales de creación de una FAV6.

\section{Objetivo}

Determinar la utilidad de la ecografía doppler vascular en la sala de hemodiálisis en la identificación de pacientes portadores de un CVT susceptibles de la construcción de una FAVI.

\section{Material y Método}

Se ha efectuado un estudio observacional y transversal en personas mayores de 18 años, prevalentes en HD asistida tres veces por semana en la Unidad de Diálisis del Hospital de Mollet, durante febrero de 2017, portadores de un CVT. Para determinar la utilidad de la ecografía doppler, se realizaron evaluaciones mediante ecografía portátil modo B, por el mismo explorador, en la sala de HD, utilizando un transductor lineal de $7,5 \mathrm{MHz}$ (monitor SiteRite ${ }^{\circledR}$ ) aplicado sobre 3 puntos diferentes de ambos brazos (proximal, medio y distal), y sobre 2 puntos de ambos antebrazos (proximal y distal), recogiéndose un promedio de las determinaciones realizadas. Se recogieron parámetros morfológicos cualitativos (presencia/ ausencia de calcificación arterial) y cuantitativos (diámetro y profundidad) de la arteria radial, la arteria humeral y la vena basílica de ambas extremidades superiores. Se determinaron como criterios ecográficos determinantes de éxito en la creación de una FAV, si el paciente presentaba, además de la calidad de la pared vascular (calcificaciones), un diámetro 
mínimo $\geq 2 \mathrm{~mm}$ para la arteria nutricia (FAVn y FAVp); $\mathrm{y} \geq 2,5 \mathrm{~mm}(F A \bigvee n) 0 \geq 4,0 \mathrm{~mm}$ (FAVp) para la vena eferente ${ }^{7}$. Así mismo se recogieron variables socio-clínicas de la población a estudio. Se realizó un análisis descriptivo de las variables a estudio, describiendo las variables cuantitativas como medias \pm desviación estándar, utilizando las frecuencias absolutas y relativas para las variables categóricas.

\section{Resultados}

De las 63 personas prevalentes en HD crónica, 9 eran portadores de un CVT yugular (14\%), conformando la muestra a estudio. Las características socioclínicas de la muestra fueron: $55,6 \%$ mujeres, edad media $67,1 \pm 17,8$ años, tiempo medio en HD $10,7 \pm 11,9$ meses, índice de masa corporal medio $26,8 \pm 4,1 \mathrm{~kg} / \mathrm{m}^{2}, 55,6 \%$ diabetes como nefropatía primaria, tiempo medio del CVT actual de $9,3 \pm 12,1$ meses ( $88,9 \%$ de localización derecha).

En la Tabla 1 se muestran los valores medios de las exploraciones ecográficas (diámetro y profundidad) de los diferentes vasos explorados. En el 33,3\% de los casos se evidenció una calcificación como mínimo. En la mayoría de los casos $(66,7 \%)$ se evidenció ausencia de calcificaciones, una placa aislada de calcificación $(11,1 \%)$ y más de una $(22,2 \%)$.

Según los valores obtenidos de los parámetros morfológicos vasculares estudiados, es posible la construcción de una FAVn o una FAVp de extremidad superior en todas las personas portadoras de CVT: 3 casos $(33,3 \%)$ FAVn radiocefálica, 3 casos $(33,3 \%)$ FAVn o FAVp humerobasílica y 3 casos $(33,3 \%)$ FAVn o FAVp húmero perforante.

Tabla 1. Valores medios de las exploraciones ecográficas, diámetro y profundidad, de los diferentes vasos explorados.

\begin{tabular}{lcc}
\hline Vaso & $\begin{array}{c}\text { Diámetro en } \mathrm{mm} \\
\text { (media } \pm \text { DE) }\end{array}$ & $\begin{array}{c}\text { Profundidad en mm } \\
\text { (media } \pm \text { DE) }\end{array}$ \\
\hline Vena basílica izquierda & $5,1 \pm 0,5$ & $10,1 \pm 2,1$ \\
\hline Arteria humeral izquierda & $4,8 \pm 0,6$ & $10,5 \pm 3,3$ \\
\hline Arteria radial izquierda & $2,7 \pm 0,7$ & $6,0 \pm 1,6$ \\
\hline Vena basílica derecha & $4,3 \pm 0,6$ & $10,1 \pm 2,1$ \\
Arteria humeral derecha & $4,7 \pm 0,3$ & $9,2 \pm 2,7$ \\
\hline Arteria radial derecha & $2,6 \pm 0,4$ & $6,6 \pm 2,0$ \\
\hline
\end{tabular}

\section{Discusión}

El $14,3 \%$ de las personas prevalentes de nuestra Unidad de HD se dializaban mediante un CVT implantado a través de la vena yugular durante el período de estudio. Esta tasa de CVT registrada cumple con los estándares de calidad recomendados en las Guías de Práctica Clínica. Según la nueva Guía Clínica Española del Acceso Vascular para Hemodiálisis, el porcentaje de personas prevalentes con más de 3 meses en programa de HD que, a 31 de diciembre del año estudiado, se dializan a través de un CVT debe ser igual o inferior al $20 \%$.

A partir de los resultados obtenidos tras analizar diversos parámetros morfológicos vasculares, todas las personas portadoras de un CVT en nuestra Unidad de HD son candidatas de la construcción de una FAV en extremidades superiores tanto FAVn como FAVp. Se ha visto el diámetro de la arteria nutricia y de la vena eferente determinado antes de efectuar la anastomosis arteriovenosa puede ser predictivo de la función de la FAV-9. De esta forma, cuanto mayor es el diámetro de la vena eferente obtenido por el mapeo ecográfico prediálisis, menor es el riego de fallo de maduración y mayor es la supervivencia de la FAV ${ }^{8}$. No se recomienda la creación de una FAV cuando el diámetro preoperatorio de la vena eferente es inferior a $2,5 \mathrm{~mm}^{9}$. Con respecto a la conversión de CVT a FAV, hay que recordar el artículo clásico publicado por Asif y cols en 2005 en el cual se evidenció que a la mayoría de personas prevalentes dializadas a través de un CVT que fueron evaluados mediante exploración física y venografía $(70 / 86,81,4 \%)$ se les pudo construir con éxito una FAVn (66) o una FAVp $(4)^{10}$.

Desde hace 8 años, se recomienda incorporar el ecógrafo portátil dentro de las salas de HD ${ }^{5}$. Desde entonces, se han descrito una serie de beneficios relacionados con la introducción del ecógrafo a la sala de $\mathrm{HD1}, 6,10-14$.

1. Apoyo en las primeras punciones de la FAV.

2. Apoyo en la punción de una FAV con elevado grado de dificultad.

3. Técnica de cribado para diagnosticar precozmente la patología de la FAV.

4. Cambio del tipo de acceso vascular permanente.

5. Cambio de la técnica de punción de la FAV.

6. Incremento de la calidad asistencial.

7. Aumento de la calidad de vida de la persona en programa de HD. 
Recientemente, se ha evidenciado que es viable un cambio en la técnica de punción de la FAV (conversión de área a escalonada) a partir de la información obtenida mediante el ecógrafo portátilil. Además, el presente estudio muestra que el ecógrafo aplicado "in situ" en la sala de HD es eficaz para valorar la conversión del acceso vascular permanente utilizado (de CVT a FAV). Además de utilizar el ecógrafo en la sala de HD, también se recomienda su aplicación en la consulta externa de enfermedad renal crónica avanzada (ERCA) para efectuar el mapeo vascular pre-HD de ambas ES con el objetivo de reducir la tasa de CVT en la persona incidente $^{5}$. En este sentido, Georgiadis y cols. publicaron un metaanálisis que incluyó 5 ensayos clínicos randomizados (574 personas) con resultado favorable a utilizar el mapeo vascular ecográfico rutinario versus selectivo como complemento a la exploración física respecto a la tasa de fallo inmediato de la FAVn ${ }^{15}$.

En vista de los resultados obtenidos podemos concluir que según los valores obtenidos de los parámetros morfológicos vasculares estudiados mediante ecografía doppler, es posible la construcción de una FAVn o una FAVp de extremidades superiores en todas las personas portadoras de CVT de la muestra estudiada.

El ecógrafo portátil utilizado "in situ" en la sala de HD es una exploración de imagen útil para conseguir un cambio en el tipo de acceso vascular permanente, es decir, para la conversión de un CVT en una FAV en las personas en HD crónica.

Recibido: 8 agosto 2017

Revisado: 25 septiembre 2017

Modificado: 5 noviembre 2017

Aceptado: 15 enero 2018

\section{Bibliografía}

1. National Kidney Foundation. KDOQI Clinical Practice Guidelines and Clinical Practice Recommendations for 2006 Updates: Hemodialysis Adequacy, Peritoneal Dialysis Adequacy and Vascular Access. Am J Kidney Dis. 2006;48(Supl 1):S1-322.

2. Roca-Tey R, Arcos E, Comas J, Cao H, Tort J, Committee CR. Vascular access for incident hemodialysis patients in Catalonia: analysis of data from the Catalan Renal Registry (2000-2011). J Vasc Access. 2015;16(6):472-9.

3. Roca-Tey R, Arcos E, Comas J, Cao H, Tort J. Starting hemodialysis with catheter and mortality risk: persistent association in a competing risk analysis. $J$ Vasc Access. 2016;17(1):20-8.

4. Gruss E, Portolés J, Tato $A$, López Sánchez P, Velayos $P$, Gago MC et al. Clinical and economic repercussions of the use of tunneled haemodialysis catheters in a health area. Nefrología 2009;29(2):123-9.

5. Roca-Tey R. El acceso vascular para hemodiálisis: la asignatura pendiente. Nefrologia 2010;30:280-7.

6. Ibeas J, Roca-Tey R, Vallespín J, Moreno T, Moñux G, Martí-Monrós A et al, por el Grupo Español Multidisciplinar del Acceso Vascular (GEMAV). Guía Clínica Española de Acceso Vascular para Hemodiálisis. Enferm Nefrol 2018;21(Supl 1):S1-256.

7. Silva MB, Hobson RW, Pappas PJ, Jamil Z, Araki $C T$, Goldberg MC et al. A strategy for increasing use of autogenous hemodialysis access procedures: impact of preoperative non invasive evaluation. J Vasc Surg. 1998;27:302-7.

8. Dageforde LA, Harms KA, Feurer ID, Shaffer D. Increased minimum vein diameter on preoperative mapping with duplex ultrasound is associated with arteriovenous fistula maturation and secondary patency. J Vasc Surg. 2015;61(1):170-6.

9. Bashar K, Clarke-Moloney M, Burke PE, Kavanagh $E G$, Walsh SR. The role of venous diameter in predicting arteriovenous fistula maturation: when not to expect an AVF to mature according to pre-operative vein diameter measurements? A best evidence topic. Int J Surg. 2015;15:95-9. 
10. Asif A, Cherla G, Merrill D, Cipleu CD, Briones $P$, Pennell P. Conversion of tunneled hemodialysis catheter-consigned patients to arteriovenous fistula. Kidney Int. 2005 Jun;67(6):2399-406.

11. Hanafusa N, Noiri E, Nangaku M. Vascular Access puncture under ultrasound guidance. Ther Apher Dial. 2014 Apr;18(2):213-4.

12. Patel RA, Stern AS, Brown M, Bhatti S. Bedside UItrasonography for arteriovenous fistula cannulation. Semin Dial. 2015 Jul-Aug;28(4):433-4.

13. Darbas-Barbé R, Roca-Tey R, Cabot C, Tinto G, Balada C, Taboada MJ et al. Cambio de técnica de punción de la fístula arteriovenosa a partir de los hallazgos ecográfícos. Enferm Nefrol 2016;19(4): 366-71.

14. Granados Navarrete I, Ibeas López JA, Iglesias Sanjuán R, Mañé Buixó N, Ramírez Prat N, Rodríguez Moreno EM et al. Enfermería en la detección precoz de patologia subyacente y punción ecodirigida en el acceso vascular problemático a través de la integración de la ecografia portátil in situ. Libro de comunicaciones XXV Congreso Nacional de la Sociedad Española de Enfermería Nefrológica (SEDEN). Madrid: SEDEN;2016. p. 164-73.

15. Georgiadis GS, Charalampidis DG, Argyriou C, Georgakarakos EI, Lazarides MK. The Necessity for Routine Pre-operative Ultrasound Mapping Before Arteriovenous Fistula Creation: A Meta-analysis. Eur J Vasc Endovasc Surg. 2015;49(5):600-5.

Este artículo se distribuye bajo una Licencia Creative Commons Atribución-NoComercial 4.0 Internacional. https://creativecommons.org/licenses/by-nc/4.0/

Open Access (c) (;) (8) 